\title{
Short-time dynamics of colloidal suspensions
}

\author{
C. P. Lowe* \\ Computational Physics, Faculty of Applied Physics, Delft University of Technology, Lorentzweg 1, 2628 CJ Delft, The Netherlands \\ D. Frenkel
}

FOM Institute for Atomic and Molecular Physics, Kruislaan 407, 1098 SJ Amsterdam, The Netherlands

(Received 16 February 1996)

\begin{abstract}
We report numerical simulations of the velocity autocorrelation function (VACF) for tagged particle motion in a colloidal suspension. We find that the asymptotic decay follows the theoretical expression for the VACF of an isolated particle, but with the suspension viscosity replacing the pure fluid viscosity (at long times the suspension behaves, so far as a tagged particle is concerned, like a fluid with the suspension viscosity-as an "effective fluid"). While physically appealing, this observation is hard to reconcile with a recent theoretical prediction that at long times the VACF in a suspension should be the same as the VACF at infinite dilution. It also differs, in a rather subtle manner, from a scaling rule which has been used in the analysis of experimental and computer simulation results. From the scaling behavior of the VACF we conclude that effective fluid behavior only occurs on a time scale somewhat longer than the time taken for transverse momentum to diffuse a particle radius. This contrasts with the findings of earlier workers who concluded that effective fluid behavior is already observed at much shorter times. [S1063-651X(96)03909-8]
\end{abstract}

PACS number(s): 82.70.Dd, 05.40.+j, 66.20.+d, 82.20.Wt

\section{INTRODUCTION}

A colloidal suspension can be considered as a mixture in which the particles of one component are much bigger than those of all the others. If the size difference is sufficiently large, the smaller components can be thought of as forming fluid which occupies the space between the big particles. The larger particles are often referred to as Brownian particles, named after the botanist Robert Brown, who was the first to observe the apparently random motion of small particles suspended in water. Starting with Brown's observation, our understanding of these systems has evolved in an interesting series of historical jumps. It was not until over seventy years later that Einstein [1] proposed that the motion of the Brownian particles (Brownian motion) arose from the accumulated effects of individual collisions between Brownian particles and the particles of the host fluid. This was strong evidence that fluids were molecular in nature, a view not universally accepted at that time. Einstein was also able to make progress with the theory of transport processes in Brownian suspensions. First he derived the expression for the diffusion coefficient $D_{0}$ of a spherical particle in the dilute limit [2]

$$
D_{0}=\frac{k T}{6 \pi \eta_{0} a}
$$

where $a$ is the particle radius and $\eta_{0}$ is the shear viscosity of the solvent. This is known as the Stokes-Einstein diffusion coefficient. Secondly [3], Einstein derived the first order correction to the ratio of suspension viscosity $\eta_{\phi}$ to the solvent viscosity,

\footnotetext{
* Present address: FOM Institute for Atomic and Molecular Physics, Kruislaan 407, 1098 SJ Amsterdam, The Netherlands.
}

$$
\frac{\eta_{\phi}}{\eta_{0}}=1+\frac{5}{2} \phi+O\left(\phi^{2}\right)
$$

where $\phi$ is the proportion of space occupied by the Brownian particles.

Knowledge of the diffusion coefficient allows us to describe the behavior of Brownian particles on a time scale long compared to the time it takes velocity correlations to decay. If one assumes that the motion of the Brownian particle can be modeled as a Markovian process, i.e., that a random force acts on the particle at one instant which is not correlated with the random force at any previous instant, one can use a classical Langevin equation to describe Brownian motion [4]. By definition the velocity autocorrelation function in $d$ dimensions $C_{v}(t)$ is given by

$$
C_{v}(t)=\frac{1}{d}\langle\mathbf{v}(0) \cdot \mathbf{v}(t)\rangle
$$

where $\mathbf{v}(t)$ is the particle velocity. The Langevin approach predicts an exponential decay for the velocity autocorrelation function (VACF), which, in three dimensions, has the form

$$
C_{v}(t)=C_{v}(0) \exp \left(-\frac{9 \tau_{0}}{2 \rho^{*}}\right),
$$

where $\rho^{*}$ is the ratio of the particle density to the fluid density, $C_{v}(0)$ the initial value of the VACF, and $\tau_{0}$ a dimensionless time parameter. This time parameter is defined by $\tau_{0}=\nu t / a^{2}$, where $\nu\left(=\eta_{0} / \rho\right)$ is the kinematic viscosity. The velocity autocorrelation function can in turn be related to a more macroscopically observable quantity, the mean-square displacement $\Delta(t)$,

$$
\frac{\Delta(t)}{2 d t}=\int_{0}^{t} C_{v}\left(t^{\prime}\right) d t^{\prime}-\frac{1}{t} \int_{0}^{t} t^{\prime} C_{v}\left(t^{\prime}\right) d t^{\prime} .
$$


At long times the second term (almost always) goes to zero, so by making use of the Einstein definition of the diffusion coefficient we have

$$
D=\lim _{t \rightarrow \infty} \frac{\Delta(t)}{2 d t}=\int_{0}^{\infty} C_{v}\left(t^{\prime}\right) d t^{\prime} .
$$

Clearly, as long as the time integral of the VACF is not a constant, it makes no sense to describe the motion of a particle as diffusive. The Langevin result implies that this time, which we denote $\tau_{v}$, should be of the order $\tau_{v} \sim \rho^{*} \nu / a^{2}$. However, when Alder and Wainwright [5] studied the decay of the VACF in a hard-sphere fluid they found that rather than decaying exponentially, as the Markovian theory predicts, it had a surprisingly slow algebraic decay of the form $C_{v}(t) \sim t^{-d / 2}$. Alder and Wainwright also provided the explanation for what was to become known as the "long-time tail." Roughly speaking, momentum is conserved in the fluid so any momentum transferred to the fluid by the particle cannot just disappear. Part of it diffuses slowly away (the remainder is rapidly carried off by the propagation of sound waves). While this is happening the particle feels an additional push from the fluid in its original direction of motion which in turn gives rise to the slow decay of the VACF. Consequently, the "random" force exerted by the fluid on a Brownian particle is not quite as random as one might have imagined, it actually depends on what was happening to the particle long ago - the process is non-Markovian. Although Alder and Wainwright originally considered a hard-sphere fluid, the same argument is not only quantitatively correct but also quantitatively much more important for a colloidal suspension. In the years since their discovery, long-time tails have developed into something of a cottage industry. The theory was recast more formally in terms of mode coupling [6] and kinetic theory [7] and tested both experimentally [8-11] and by computer simulation [12-16]. For a colloidal particle in an incompressible fluid Hauge and Martin-Löf [17] derived the Laplace transformed equations of motion for the entire time range. In three dimensions the explicit longtime decay of the VACF is given by

$$
C_{v, \text { long }}\left(\tau_{0}\right)=\frac{C_{v}(0) \rho^{*}}{9 \sqrt{\pi}} \tau_{0}^{-3 / 2}
$$

Again these results have been verified by experiment [18] and computer simulation [14].

The existence of long-time tails may seem interesting, if a little esoteric. However, they have important practical consequences. For instance, the presence of the long-time tail means that the diffusion coefficient in quasi-two-dimensional fluids, such as free-standing films, does not exist. Another consequence, of direct relevance for the present study, is that purely diffusive behavior of a colloidal particle only occurs on a time scale that is several orders of magnitude longer than one would expect from the Langevin equation, i.e., $\tau_{v}$ $\gg \rho^{*} a^{2} / \nu$ (this is clearly illustrated by the experimental results of Zhu et al. [18] for instance).

Although long-time tails greatly extend the time scale over which velocity correlations influence Brownian motion, generally speaking there is still a convenient separation of time scales between the transient effects of the VACF and the time scale on which the particles significantly change their positions. The time scale for particle displacement $\tau_{p}$ can be characterized by the time taken for a particle to diffuse a distance of its own radius $\tau_{p}=a^{2} / D_{0}$. Making use of the Stokes-Einstein relation, Eq. (1), gives us $\tau_{p}$ $=9 m \nu / 2 \rho^{*} k T$. For particles dispersed in a waterlike fluid at room temperature this implies that the condition $\tau_{p} \gg \tau_{v}$ is still reasonably well satisfied, even allowing for the effect of the long-time tail, for particles down to about $1 \mu \mathrm{m}$. For times $t<\tau_{p}$, the regime of "short-time" dynamics, transport coefficients can be calculated assuming that the configuration is essentially fixed. Within this approximation considerable progress has been made in deriving higher order terms in the "Virial expansion" of both the short-time single-particle diffusion coefficient [19] and the short-time suspension viscosity [20]. With advances in experimental techniques it is now possible to study the dynamics of Brownian particles on these short-time scales and, indeed, the experimental [21], theoretical [19], and numerical [22] results, particularly for the VACF, all seem to agree quite nicely. In this article we consider the short-time regime. Hence where, in the remainder of this paper, we talk about "long-time"' behavior we are always referring to times for which $\tau_{0}$, although greater than unity, is still much less than $\tau_{p}$.

In recent experiments $[18,23]$ the transient behavior of the mean-square displacement was studied at very short times, giving detailed information about how the asymptotic regime is reached. These studies have provided another surprise. Although there is a strong analogy between colloidal suspensions and simple fluids, there is at least one way in which they should fundamentally differ. The interactions between particles, which are after all the reason that transport coefficients in a concentrated suspensions differ from their values in the dilute limit, are of a different nature. Whereas intermolecular forces between individual molecules are essentially instantaneous, colloidal particles also influence one another via the fluid occupying the space between them. The speed at which these interactions propagate depends on the properties of the fluid itself. As "hydrodynamic" interactions between particles develop by the propagation of momentum through the fluid, they were generally assumed to develop on a time scale determined by the kinematic viscosity of the fluid, i.e., $\tau_{0} \sim 1$. However, by comparing the shorttime dynamics of particles in a concentrated suspension with those of an isolated particle, recent experiments [18,23] found no evidence for such a time lag. In fact, it was found that the two deviated even at the shortest times that could be measured $\left(\tau_{0}<1\right)$. Zhu et al. [18] also found that all the experimental data could be collapsed onto the single-particle curve if the time was rescaled in units of $a^{2} / \nu_{\phi}$, where $\nu_{\phi}$ is the kinematic viscosity of the suspension. The conclusion was, that even on time scales where $\tau_{0}\left(\equiv t \nu / a^{2}\right)<<1$, a colloidal suspension behaves like an "effective fluid," by which we mean a fluid with the viscosity of the suspension. This is surprising because, if the hydrodynamic interactions develop on a time scale $\tau_{0} \sim 1$, then one would expect that the suspension can only behave as an effective fluid on time scales $\tau_{0} \gg 1$. Experiments performed by Kao, Yodh, and Pine [23] probed $D(t)$ on very short time scales, down to $\tau_{0}<0.1$, and indicated that the same scaling worked in this time regime. However, in Ref. [23] it was pointed out that 
other scalings could perform equally well. Finally, computer simulations performed by Ladd $[14,24]$ also seemed to confirm that the scaling of Ref. [18] is indeed obeyed at very short times. One possible explanation has been proposed by Español, Rubio, and Zúñiga [25,26] who suggested that the speed of sound and not the kinematic viscosity determines the time scale at which the hydrodynamic interactions propagate. In a typical colloidal suspension, sound wave propagation is several orders of magnitude faster than vorticity diffusion. This, they suggested, could make effective-fluid behavior possible in the very short-time regime.

A second topic that we address in this paper is the longtime behavior of the VACF in concentrated suspensions. Although the result for the VACF of a single particle is well known, there remain contradictory theoretical results for the VACF in a concentrated suspension. A calculation performed by Milner and Liu [27] suggested that the effectivefluid argument is correct, at least to order $\phi$ in the volume fraction (they further speculated that it was generally true). On the other hand Cichocki and Felderhof $[28,29]$ argued that, again to order $\phi$, the long-time form of the VACF for a particle in a concentrated suspension should be identical to the result at infinite dilution. As Cichoki and Felderhof pointed out, this result is hard to reconcile with the scaling that is reported in experiments and computer simulations. The result of Ref. [29] is also somewhat counterintuitive, because it implies that, at long times, a particle no longer experiences the presence of its neighbors. Cichocki and Felderhof suggested [29] that the experimental results covered times too short for the asymptotic long-time tail to adequately describe the VACF. Yet, the data obtained by Zhu et al. [18] did at least extend to times long enough for them to clearly observe the long-time tail in the dilute limit.

In light of these observations and theories we have performed computer simulations in an attempt to clarify the issues raised. First we wish to examine more closely the scaling proposed by Zhu et al. [18] and what it implies for the velocity autocorrelation function. This leads us to propose a slightly different scaling. Finally we describe numerical results for the VACF of a Brownian particle and study both the short- and long-time behavior in a concentrated suspension.

\section{THE SCALING OF THE VELOCITY AUTOCORRELATION FUNCTION}

The VACF for an isolated Brownian particle (see for instance the results derived by Hauge and Martin-Löf [17]) depends on the same three parameters as the Langevin result [Eq. (4)]. To recapitulate, these parameters are the initial value of the VACF $C_{v}(0)$ the density ratio $\rho^{*}$, and the reduced time $\tau_{0}$. In reality $\rho^{*}$ is a parameter which cannot differ much from unity-otherwise the colloid would be unstable-so we consider it fixed. Having done so, the VACF becomes a unique function of $\tau_{0}$ and $C_{v}(0)$, which is in principle known. Even more simple is the normalized VACF $C_{v}(t) / C_{v}(0)$ that becomes a function of $\tau_{0}$ only. We shall call this function $f\left(\tau_{0}\right)$.

The scaling behavior of the mean-square displacement is not usually discussed in terms of the velocity autocorrelation function. It is more usual to consider the time-dependent diffusion coefficient $D(t)$, defined as $D(t)=\Delta(t) / 2 d t$. The two functions are, however, related by Eq. (5), so any scaling which applies to $D(t)$ also corresponds to some scaling for $C_{v}(t)$. It is therefore natural to consider what the scaling for $D(t)$ applied by Zhu et al. [18] would imply for the scaling of the VACF. First, we consider a particle in the dilute limit and assume that we have measured $D(t)$. We then apply the scaling of Ref. [18] to collapse our data onto a single curve. To this end we first scale the time from $t$ to $\tau_{0}$ to give us $D\left(\tau_{0}\right)$. This corresponds to scaling the time for the VACF from $t$ to $\tau_{0}$ and multiplying $C_{v}(t)$ by $a^{2} / \nu$ (the multiplicative factor enters because scaling the time should not change the integral of the VACF). Next we divide by the diffusion coefficient at infinite dilution to yield the function $g\left(\tau_{0}\right)=D\left(\tau_{0}\right) / D_{0}$. This step simply corresponds to dividing the VACF by $D_{0}$ so the scaled VACF $f\left(\tau_{0}\right)$ corresponding to $g\left(\tau_{0}\right)$ is simply

$$
f\left(\tau_{0}\right)=\frac{a^{2} C_{v}\left(\tau_{0}\right)}{\nu D_{0}}
$$

which, if we substitute the Stokes-Einstein result for $D_{0}$, becomes

$$
f\left(\tau_{0}\right)=\frac{9}{2} \frac{C_{v}\left(\tau_{0}\right)}{C_{v}(0)},
$$

where we have also set $\rho^{*}=1$. The scaled normalized VACF is therefore only a function of $\tau_{0}$ as expected.

What Zhu et al. did was to extend this argument to a concentrated suspension by defining $\tau_{0}$ in terms of the suspension viscosity $\nu_{\phi}$. We define this reduced time $\tau_{0, \phi}$, to distinguish it from the reduced time defined in terms of the solvent viscosity $\nu_{0}$. They then followed a similar procedure-rescaling the time from $t$ to $\tau_{0, \phi}$ and dividing by $D_{\phi}$, where $D_{\phi}$ is the diffusion coefficient in the suspension-to give the scaled function $g_{\phi}\left(\tau_{0, \phi}\right)\left[=D\left(\tau_{0, \phi}\right) / D_{\phi}\right]$. In the experiments, this function was found to be equal to the single-particle function $g\left(\tau_{0}\right)$. Following the same argument as before, the scaled VACF $f_{\phi}\left(\tau_{0, \phi}\right)$ corresponding to $g_{\phi}\left(\tau_{0, \phi}\right)$ is now

$$
f_{\phi}\left(\tau_{0, \phi}\right)=\frac{a^{2} C_{v, \phi}\left(\tau_{0, \phi}\right)}{\nu_{\phi} D_{\phi}},
$$

where $\nu_{\phi}$ is the suspension viscosity and $C_{v, \phi}(t)$ the VACF in the concentrated suspension. However, if we now try to eliminate $D_{\phi}$ we have a problem because the Stokes-Einstein equation is not valid for a suspension (at least on the time scale we are considering here). By introducing $D_{S E, \phi}$, the Stokes-Einstein diffusion coefficient for a single particle in a fluid with the suspension viscosity, we can write

$$
f_{\phi}\left(\tau_{0, \phi}\right)=\frac{a^{2} C_{v, \phi}\left(\tau_{0, \phi}\right)}{\nu_{\phi} D_{S E, \phi}} \frac{D_{S E, \phi}}{D_{\phi}},
$$

which, following from the Stokes-Einstein equation, gives

$$
f_{\phi}\left(\tau_{0, \phi}\right)=\frac{9}{2} \frac{C_{v, \phi}\left(\tau_{0, \phi}\right)}{C_{v}(0)} \frac{D_{S E, \phi}}{D_{\phi}} .
$$


The scaling Zhu et al. observed $g_{\phi}\left(\tau_{0, \phi}\right)=g\left(\tau_{0}\right)$ is equivalent to saying $f_{\phi}\left(\tau_{0, \phi}\right)=f\left(\tau_{0}\right)$, which in turn implies that $C_{v, \phi}\left(\tau_{0, \phi}\right)=\left(D_{\phi} / D_{S E, \phi}\right) C_{v}\left(\tau_{0}\right)$. The implication is that the VACF looks like the single-particle VACF for a particle in a fluid with the suspension viscosity but multiplied by a factor of $D_{\phi} / D_{S E, \phi}$. If $D_{\phi} / D_{S E, \phi}$ is equal to unity the scaling implies effective-fluid behavior, otherwise it does not. But, as we mentioned before, the Stokes-Einstein equation does not yield the correct short-time diffusion coefficient for a suspension of colloidal spheres, and hence $D_{\phi} / D_{S E, \phi} \neq 1$.

It is important to note that if the suspension behaves like an effective fluid, the above scaling will almost work. For an isolated particle we know that at long times $f\left(\tau_{0}\right) \sim \tau_{0}{ }^{-3 / 2}$. If we suppose for a moment that the effective-fluid argument is correct, then at long times the scaling proposed by Zhu et al. will work if an "apparent" viscosity $\nu_{\text {eff }}$ given by

$$
\nu_{e f f}=\nu_{\phi}\left(\frac{D_{\phi}}{D_{S E, \phi}}\right)^{2 / 3}=\nu_{\phi} \alpha^{2 / 3}
$$

is used to define $\tau_{0, \phi}$ instead of the suspension viscosity $\nu_{\phi}$. This makes the derivatives of the scaled function identical to those of the single-particle function and, since both functions are approaching the same asymptote, they will coincide at long times. Because the value of $\alpha$ is close to unity [22], the apparent viscosity needed to make the functions coincide at long times only differs from the suspension viscosity by a small factor (ranging from $2 \%$ at $\phi=0.05$ to $14 \%$ at $\phi=0.30$ ). Such a small effect may well be compatible with the uncertainty in the experimental data and is rather unimportant. However, at short times the two functions will differ if either the effective-fluid assumption breaks down or the single-particle curve is no longer adequately described by the long-time tail. This would make it difficult to draw any definite conclusions about the onset of effective-fluid behavior.

Before we go on to describe our numerical simulation, we would like to suggest a more appropriate scaling for $D(t)$ in an effective fluid. We mentioned earlier that applying the isolated particle scaling to particles in a suspension leads to an inconsistency [see Eq. (12)]. The fact that $D_{\phi}$ is not equal to $D_{S E, \phi}$ means that it is impossible to scale $D(t)$ in a suspension onto the single-particle curve over all times. We therefore propose a slightly different scaling which cures this problem by using an additive, rather than a multiplicative, constant

$$
g_{\phi}\left(\tau_{0, \phi}\right)=\frac{D\left(\tau_{0, \phi}\right)}{D_{S E, \phi}}+\left(1-\frac{D_{\phi}}{D_{S E, \phi}}\right) .
$$

Adding a constant to $D\left(\tau_{0, \phi}\right)$ is equivalent to the assumption that (at long times) the VACF in a concentrated suspension looks like the scaled single-particle function. The short-time deviation from effective-fluid behavior integrates to a constant offset in $g_{\phi}\left(\tau_{0, \phi}\right)$. Effective-fluid behavior sets in when this deviation has decayed to zero. It is in this regime that the above scaling should hold. This is discussed in more detail in the Appendix.

\section{DESCRIPTION OF THE MODEL}

We have used a hybrid hard-sphere, lattice-gas model to simulate suspensions. The initial configurations are generated by a Monte Carlo simulation of a hard-sphere fluid. In keeping with our assumption of short-time dynamics, we impose the time-scale separation $\tau_{v} \ll \tau_{p}$, so the positions of the colloidal particles do not change during a run. The timedependent hydrodynamic interactions between the spheres are computed by embedding them in a simple model fluid, namely, a lattice gas simulated at the Boltzmann level. The lattice-Boltzmann model is a preaveraged version of a lattice-gas cellular automaton (LGCA) model of a fluid of the type introduced by Frisch, Hasslacher, and Pomeau [30]. In lattice-gas cellular automata the state of the fluid at any (discrete) time is specified by the number of particles at every lattice site and their velocity. Particles can only move in a limited number of directions (towards neighboring lattice points) and there can be at most one particle moving on a given "link." The time evolution of the LGCA consists of two steps; propagation, during which every particle moves one time step along its link to the next lattice site, and collision, where at every lattice site particles can change their velocities by collision (subject to the condition that these collisions conserve the number of particles and momentum and retain the full symmetry of the lattice). In the latticeBoltzmann method (see, e.g., [31]) the state of the fluid system is no longer characterized by the number of particles that move in direction $\mathbf{c}_{i}$ on lattice site $\mathbf{r}$, but by the probability of finding such a particle. The single-particle distribution function $n_{i}(\mathbf{r}, t)$, describes the average number of particles at a particular node of the lattice $\mathbf{r}$, at a time $t$, with the discrete velocity $\mathbf{c}_{i}$. The hydrodynamic fields, mass density $\rho$, momentum density $\mathbf{j}$, and the momentum flux density $\boldsymbol{\Pi}$ are simply moments of this velocity distribution:

$$
\rho=\sum_{i} n_{i}, \quad \mathbf{j}=\sum_{i} n_{i} \mathbf{c}_{i}, \quad \boldsymbol{\Pi}=\sum_{i} n_{i} \mathbf{c}_{i} \mathbf{c}_{i} .
$$

The lattice model used in this work is the four-dimensional (4D) face-centered hypercubic (FCHC) lattice. A two- or three-dimensional model can then be obtained by projection in the number of required dimensions. This FCHC model is used because three-dimensional cubic lattices do not have a high enough symmetry to ensure that the hydrodynamic transport coefficients are isotropic. The time evolution of the distribution functions $n_{i}$ is described by the discretized analogue of the Boltzmann equation [32]

$$
n_{i}\left(\mathbf{r}+\mathbf{c}_{i}, t+1\right)=n_{i}(\mathbf{r}, t)+\Delta_{i}(\mathbf{r}, t),
$$

where $\Delta_{i}$ is the change in $n_{i}$ due to instantaneous molecular collisions at the lattice nodes. The postcollision distribution $n_{i}+\Delta_{i}$ is propagated in the direction of the velocity vector $\mathbf{c}_{i}$. A complete description of the collision process is given in [33]. The main effect of the collision operator $\Delta_{i}(\mathbf{r}, t)$ is to (partially) relax the shear stress at every lattice site. The rate of stress relaxation, or equivalently, the kinematic viscosity $\nu$, can be chosen almost freely.

The motion of the colloidal particle is determined by the force and torque exerted on it by the fluid. These are in turn a result of the stick boundary conditions applied at the solid- 
fluid interface. For a stationary boundary a simple bounceback rule performed on boundary links enforces the stick boundary condition. Boundary links are links connecting lattice sites inside and outside the solid object. Stick boundary conditions with a moving boundary can be performed using the Boltzmann analogue [33] of a scheme originally used for lattice gases [34]. For a moving boundary the bounce-back rule is still applied but some of the particles moving in the same direction as the solid object are allowed to "leak" through, thus matching the fluid velocity to the object velocity at the boundary. The equations of motion of the colloidal particle are integrated according to a rule [16] whereby the force and torque which act on an object give the same new velocities for both particle and fluid. This method gives us considerable flexibility in choosing the density ratio, so for our simulations we chose $\rho^{*}=1$ - a good approximation to the value one would expect in an experimental setup.

Simulating the lattice gas at the Boltzmann level has a number of advantages over the LGCA approach, for instance, the model is Gallilean invariant whereas a lattice gas is not. However, as a result of the ensemble averaging all spontaneous fluctuations in the fluid disappear, i.e., the lattice-Boltzmann model is purely dissipative. In the absence of any externally imposed fluctuations a Brownian particle in a Boltzmann fluid just sits there and does nothing. Fluctuations can be incorporated in the lattice-Boltzmann model by adding a suitable random noise term to the stress tensor [24], but we have chosen a slightly different approach. We make use of Onsager's regression hypothesis and watch the decay of a single fluctuation which we impose on the (otherwise) purely dissipative system. A similar approach was used to calculate the stress-stress autocorrelation function in Ref. [15]. This has the advantage of not adding any noise to the system, although we still need to average over different configurations. Our procedure is as follows. We take a configuration of hard spheres in a stationary fluid and assign each one a velocity taken from a Maxwell distribution. We then calculate the VACF for each particle as its velocity decays and finally we average the function over all particles. One small point is that we do not constrain the total momentum in the system to be zero because this introduces an artificial anticorrelation between the velocity of an individual particle and the velocities of its neighbors. However, since we have a net momentum in the system the VACF does not decay to zero. To correct for this we perform the calculation in the frame of reference where the total fluid momentum remains zero, so the correlation function we actually calculate is

$$
C_{v}(t)=\frac{1}{d}\langle\Delta \mathbf{v}(0) \cdot \mathbf{\Delta} \mathbf{v}(t)\rangle,
$$

where $\Delta \mathbf{v}(t)$ is the velocity of the particle relative to the velocity of the fluid. If the total momentum of the colloidal particles at a given time is $\mathbf{p}(t)$ then the total momentum gained by the fluid at that time $\boldsymbol{\Delta p}(t)$ is just $\mathbf{p}(t=0)-\mathbf{p}(t)$. The velocity of a particle relative to the fluid velocity $\boldsymbol{\Delta} \mathbf{v}(t)$ is therefore equal to $\mathbf{v}(t)-\boldsymbol{\Delta} \mathbf{p}(t) /(V \rho)$, where $\rho$ is the density of the fluid and $V$ the volume of the system. This procedure gives a VACF which, starting from a system with net momentum, decays to zero and is independent of system size (up to the time required for momentum to cross the simulation box).

\section{RESULTS}

The simulations were performed using two different sets of parameters. It is convenient to define these parameters in terms of lattice units, such that the lattice spacing, time step, and particle mass are all equal to unity. For low densities $(0.05 \leqslant \phi \leqslant 0.2)$ we used a sphere of radius 2.5 in a fluid of kinematic viscosity $1 / 6$ and fluid density 24 . For higher volume fractions $(0.20 \leqslant \phi \leqslant 0.30)$ we used a sphere of radius 4.5 in a fluid of kinematic viscosity $1 / 2$ and fluid density 24 . Using a larger representation of a sphere gives a better approximation to the hydrodynamic interactions at small particle separations. One might expect this to become important at high densities which is why we chose to switch to the larger particles (although the results obtained for $\phi=0.2$, where we tried both representations, were very similar). There is an approximation involved in mapping a sphere onto the lattice which means that the object we end up with looks like a sphere with a slightly different radius $a^{*}$ compared to the nominal radius $a$. The effective radius also depends on the fluid viscosity. This is discussed by Ladd in reference [14]. We calculated the effective radii of the spheres by calculating the VACF of an isolated particle in a fluid with the appropriate viscosity, and integrating it to obtain $D_{0}$. We then took the values of $D_{0}$ along with the mass and viscosity and used the Stokes-Einstein equation [Eq. (1)] to define the effective radii $a^{*}$. The values we obtained were 2.46 and 4.20 for spheres of nominal radius 2.5 and 4.5 , respectively. For all the calculations we were careful to eliminate the effects of the periodic boundary conditions (which are large) by only calculating the VACF up to times less than the time taken for a sound wave to cross the simulation box. For calculations at non-negligible volume fraction this time was significantly reduced because the speed of sound increases with volume fraction. The box was typically of length 150 and the speed of sound in the pure fluid was always equal to $1 / \sqrt{2}$.

First we considered the time scale on which the effects of the hydrodynamic interactions could be seen. To do this we calculated the VACF for a single particle and then the VACF for particles in a low concentration suspension $(\phi=0.01)$. In Fig. 1 we have plotted the (absolute) percentage difference between the two VACF's in terms of the reduced time $\tau_{0}$. It is clear that the difference begins to appear at the shortest times we can calculate and certainly for times $\tau_{0} \ll 1$. This is the same kind of behavior observed experimentally by Zhu et al. [18] and by Kao, Yodh, and Pine [23], although we are looking at shorter times and lower volume fractions (our volume fraction here is actually lower than that typically used experimentally as the infinite dilution result). So, our simulations confirm that the hydrodynamic interactions manifest themselves at times very short compared to the time taken for transverse momentum to be transferred, a typical interparticle separation. This in itself does not mean that some faster mechanism is required. There are always particles at very small separations, which can interact quickly, even in extremely dilute systems. To see if interactions de- 


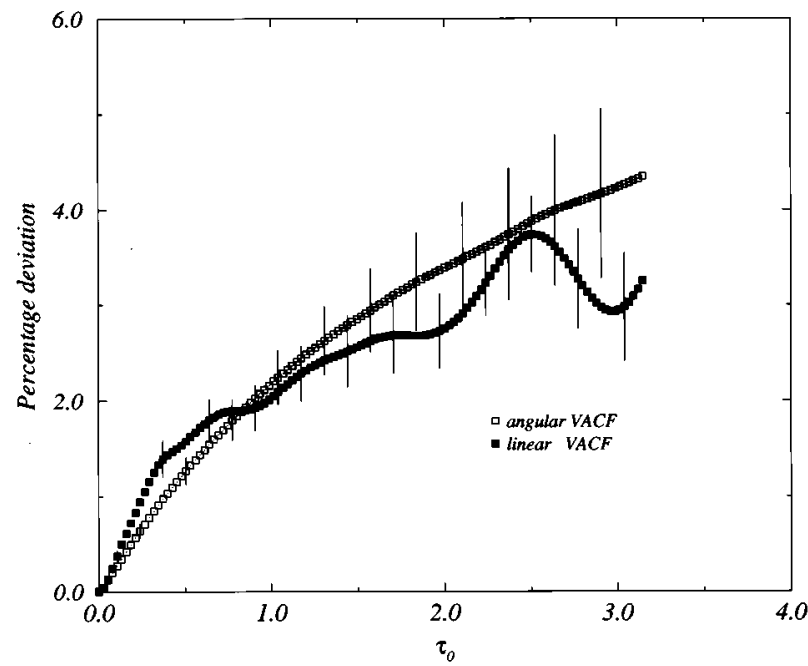

FIG. 1. The percentage deviation of the linear and angular velocity autocorrelation functions in a suspension from their values in the dilute limit, plotted as a function of the reduced time $\tau_{0}$. The volume fraction of colloidal particles in the suspension was $\phi=0.01$.

veloping by sound wave propagation are necessarily responsible for this observation, we performed an identical calculation for the angular velocity autocorrelation function (defined in the same way as the VACF but replacing the translational with the rotational velocity). In contrast to a linear velocity fluctuation, a rotational velocity fluctuation produces no sound wave, so the speed at which hydrodynamic torques between particles develop cannot depend on sound wave propagation. Our results for the angular VACF are also plotted in Fig. 1. Clearly the angular VACF behaves in the same way as the linear VACF, the hydrodynamic interactions begin to influence the decay at very short times. We know that sound wave propagation is unimportant in this case so we can conclude that it is not needed to explain the behavior we observe for the VACF.

Next we look at the form of the decay of the VACF in a concentrated suspension. In Fig. 2 we have plotted the velocity autocorrelation function divided by the theoretical long-time decay for a particle in the dilute limit [given in Eq. 7)]. For the single particle the curve appears to be approaching unity as we would expect. If we extrapolate to infinite times by fitting $C_{v}\left(\tau_{0}\right) / C_{v}$, long $\left(\tau_{0}\right)$ to a polynomial of the form $a+b \tau_{0}^{-1}+c \tau_{0}^{-2}$ then we obtain asymptotic values of $1.006 \pm 0.01$ and $0.994 \pm 0.01$ for the spheres of nominal radius 2.5 and 4.5 , respectively. This extrapolation procedure is illustrated in Ref. [16] and the quality of the extrapolation here is comparable. Having established that all is well for the single particle we now wish to consider the other curves in Fig. 2, those obtained for non-negligible volume fractions. First, the plots are approaching an asymptote, indicating that the $t^{-3 / 2}$ tail is still present in the concentrated suspensions. In Fig. 2 we have also plotted the asymptotic values expected if the long-time decay is given by Eq. (7) but with the suspension viscosity replacing the pure fluid viscosity, i.e., if the suspension behaves like an effective fluid. The values for the suspension viscosity as a function of volume fraction were taken from Ref. [22]. Again, the curves appear to be approaching the correct asymptote. At low volume fractions

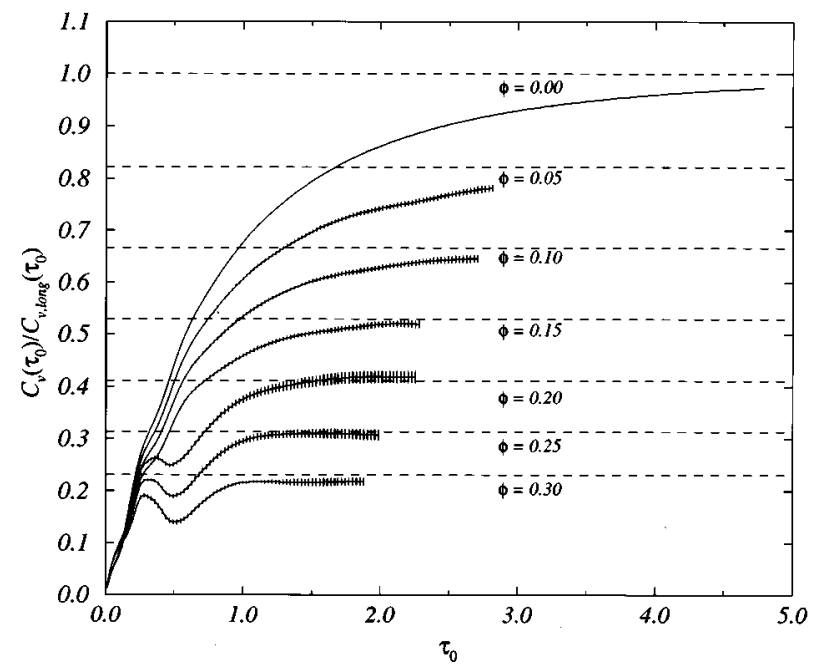

FIG. 2. The velocity autocorrelation function $C_{v}\left(\tau_{0}\right)$ divided by the theoretical long-time result $C_{v, \text { long }}\left(\tau_{0}\right)$, which is defined in the text. The results were obtained at variou volume fractions $\phi$; the solid line with no error bars, labeled $\phi=0$, is the single-particle result. The dashed lines are the asymptotic values of $C_{v}\left(\tau_{0}\right) / C_{v, \text { long }}\left(\tau_{0}\right)$ one obtains by replacing the fluid viscosity with the suspension viscosity in the equation for the long-time tail.

$(0.05,0.10$, and 0.15$)$, where the asymptotic value has not been reached during the simulation, we applied the same extrapolation procedure described above. At higher volume fractions $(0.2,0.25$, and 0.30$)$ the data has, to within the statistical errors, reached a plateau. In this case any extrapolation would of course be meaningless so we took the asymptotic value to be the plateau value. Armed with the longtime form of the VACF we made the effective fluid assumption and used Eq. (7) to convert them into viscosities. The values we obtained are plotted in Fig. 3. For completeness we have also plotted values of $D_{\phi} / D_{0}$ obtained by integrating the VACF. Clearly both sets of values are in agreement with those calculated by Ladd [22]. Our results, therefore, strongly suggest that in a concentrated suspension the VACF at long times looks like that of a single particle in a fluid with the suspension viscosity-just as predicted theoretically by Milner and Liu [27]. This result may seem unsurprising, but we recall that it contradicts the theory of Cichocki and Felderhof [28,29], according to which all curves in Fig. 2 approach the single-particle asymptote. It also differs slightly from the scaling proposed by Zhu et al. [18] which would have the asymptotes deviating from the effective-fluid asymptote by a factor of $D_{\phi} / D_{S E, \phi}$, for which we see no evidence.

Our simulations suggest that the scaled function $C_{v}\left(\tau_{\phi}\right)$ is identical to the single-particle function $C_{v}\left(\tau_{0}\right)$ at long times (we have shortened the notation for the time scale defined in terms of the suspension viscosity to $\tau_{\phi}$ ). We also know that at short times the two functions must differ because they have different integrals. The question now is, after how long do the two coincide or, equivalently, when does the suspension start to behave like an effective fluid? To answer this question we have plotted $C_{v}\left(\tau_{\phi}\right) / C_{v, \text { long }}\left(\tau_{\phi}\right)$ as a function of $\tau_{\phi}$. The data for the spheres with nominal radius 2.5 are plotted in Fig. 4 and for the spheres of nominal radius 4.5 in Fig. 5. Because we have an uncertainty as to the 


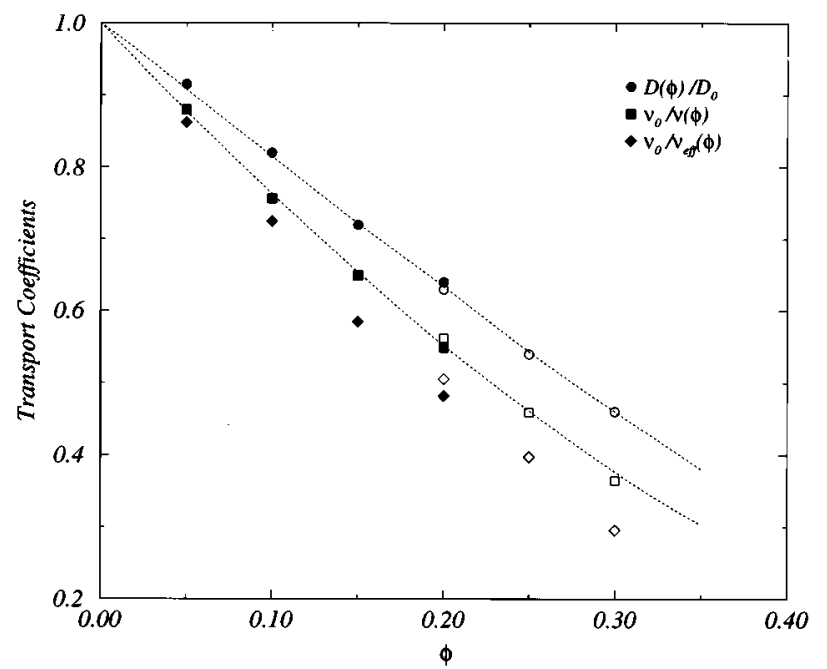

FIG. 3. Values for the transport coefficients in a colloidal suspension of volume fraction $\phi$. The circles are the diffusion coefficient $D_{\phi}$ divided by the single-particle value $D_{0}$. Values for the diffusion coefficients were calculated by integrating the velocity autocorrelation function. The squares are the solvent viscosity $\nu_{0}$ divided by the suspension viscosity $\nu_{\phi}$. Values for the suspension viscosity were calculated by assuming that the suspension behaves like an effective fluid at long times. Finally the pure fluid viscosity divided by an effective viscosity $\nu_{e f f}$. The effective viscosity was calculated by applying the scaling procedure of Zhu et al. to data for the time-dependent diffusion coefficient. Filled symbols indicate results for spheres with an effective radius of 2.46 and open symbols for spheres with an effective radius of 4.2. The solid lines are splines through the values calculated by Ladd [22].

true asymptote (or equivalently viscosity), we have scaled the data using both our upper and lower estimates for the viscosity. In Figs. 4 and 5 we show this by plotting the region bounded by these two values. Both figures suggest that VACF cannot be collapsed onto the single-particle curve for times less than those at which the single-particle curve itself reaches its asymptotic decay. The long-time tail appears to establish sooner, both literally and proportionately, in the concentrated suspensions. Remembering that our definition of effective-fluid behavior requires that these functions coincide, our simulations suggest that this is only the case for reduced times where the long-time tail adequately describes the VACF of a single particle, i.e., approximately $\tau_{\phi}>4$.

It is easy for us to look at the velocity autocorrelation function but it is a quantity that is not so easy to determine experimentally. With this in mind we have used Eq. (5) to convert our results for the VACF into time-dependent diffusion coefficients. These are plotted in Fig. 6 for the spheres with nominal radius 2.5, and in Fig. 7 for the spheres with nominal radius 4.5. We have used three scalings for the data (which has also been offset for clarity). For the uppermost data we have scaled $D(t)$ according to Eq. (14). As we expected from our analysis of the VACF, the scaling works, but only for times $\tau_{\phi}>4$. For the data in the middle of the figures we have applied the scaling proposed by Zhu et al. using values for $D_{\phi}$ and $\nu_{\phi}$ calculated from the simulation. These of course have a statistical error associated with them. The influence of these errors on the scaled function is of the order of the symbol size. Clearly the scaling does not quite

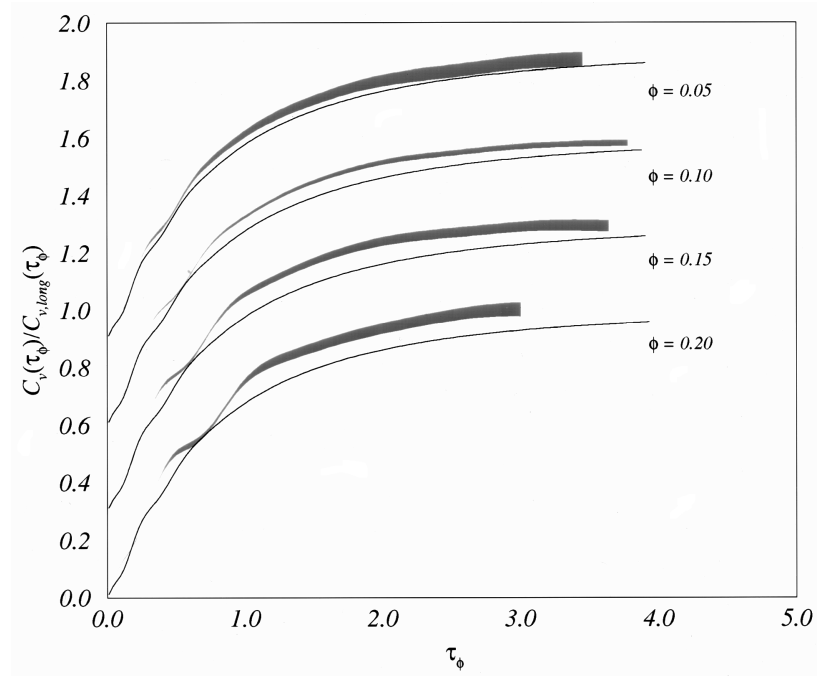

FIG. 4 . The velocity autocorrelation function $C\left(\tau_{\phi}\right)$ divided by its long-time decay $C_{v, l o n g}\left(\tau_{\phi}\right)$ (which was calculated by assuming that the suspension behaves like an effective fluid at long times). The reduced time $\tau_{\phi}$ is defined in terms of the suspension viscosity $\nu_{\phi}$ and the effective particle radius $a^{*}$ by $\tau_{\phi}=t \nu(\phi) / a^{* 2}$. These results were obtained for spheres with an effective radius of 2.46 . The solid lines are the results for the isolated particle. The gray regions are the results for non-negligible volume fractions, with volume fraction $\phi$ increasing from top to bottom. The regions are defined by our upper and lower estimates for the suspension viscosity. To assist clarity the data for volume fractions $\phi=0.05,0.10$, and 0.15 have been displaced in the $y$ direction by $0.9,0.6$, and 0.3 , respectively.

work. However, for the data at the bottom of the figures we have performed the same scaling using the value of $D_{\phi} / D_{0}$ from the simulation, but allowing the suspension viscosity to be a free parameter. By doing so we can achieve a convincing looking result but we have to use the wrong viscosity to achieve it. The apparent values for the viscosity obtained by following this procedure $\nu_{\text {eff }}$ are plotted in Fig. 3, along with the correct values. There is clearly a systematic deviation between the apparent viscosity, derived from this scaling, and the true viscosity.

\section{DISCUSSION}

By calculating the velocity autocorrelation function for a tagged particle in a colloidal suspension, we have shown that, as far as the particle is concerned, at long times the rest of the suspension behaves just like a fluid with the suspension viscosity. This we refer to as effective-fluid behavior. We have shown that although the VACF in a dilute suspension differs from the single-particle VACF at very short times, effective-fluid behavior only begins when the viscous time scale for the suspension $\tau_{\phi}$ exceeds values of the order four. From these observations, our arguments about the scaling of the mean-square displacement follow. Our data are not quite compatible with the scaling of the mean-square displacement used in Refs. [18,24]. However, the incorrect scaling for the mean-square displacement can be made to look rather compelling, even at short times, by scaling the time with a slightly incorrect viscosity. So, by just looking at the mean-square displacement it would be very easy to conclude 


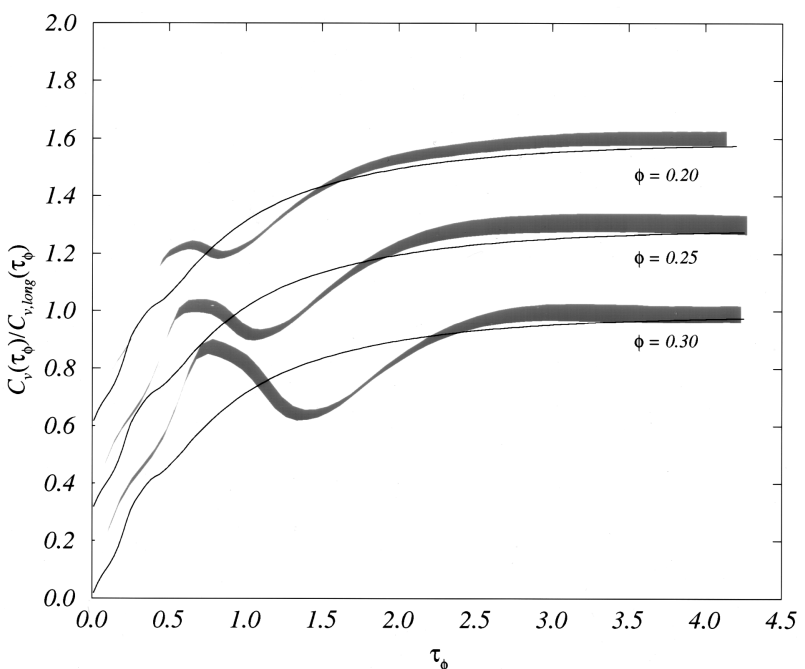

FIG. 5. The velocity autocorrelation function $C_{v}\left(\tau_{\phi}\right)$ divided by its long-time decay $C_{v, \text { long }}\left(\tau_{\phi}\right)$ (which was calculated by assuming that the suspension behaves like an effective fluid at long times). The reduced time $\tau_{\phi}$ is defined in terms of the suspension viscosity $\nu_{\phi}$ and the effective particle radius $a^{*}$ by $\tau_{\phi}=t \nu(\phi) / a^{* 2}$. These results were obtained for spheres with an effective radius of 4.20. The solid lines are the results for the isolated particle. The gray regions are the results for non-negligible volume fractions, with volume fraction $\phi$ increasing from top to bottom. The regions are defined by our upper and lower estimates for the suspension viscosity. To assist clarity the data for volume fractions $\phi=0.20$ and 0.25 and have been displaced in the $y$ direction by 0.6 and 0.3 , respectively.

that effective-fluid behavior begins at much shorter times than our results for the VACF suggest. Our results are therefore consistent with the experimental results but, by considering the VACF rather than the mean-square displacement itself, we arrived at a slightly different conclusion. However, our data are hard to reconcile with the theoretical work of Cichocki and Felderhof $[28,29]$ who stated that, to order $\phi$ in the volume fraction, the asymptotic decay of the VACF in a suspension is identical to that of an isolated particle. In contrast our simulations strongly support the conjecture of Milner and Liu - that the suspension behaves like an effective fluid at least to order $\phi$ and probably to higher orders in $\phi$.

We now wish to consider the implications of our results for the propagation of hydrodynamic interactions. We found that the effect of the hydrodynamic interactions on the VACF could be seen at very short times, certainly for $\tau_{0}$ $\ll 1$. However, we found the same behavior for the angular VACF, so this feature of the linear VACF can be explained quite simply. There are always particles at small separations which can influence each other via the diffusion of transverse momentum, even on time scales short compared to the time for the interactions to propagate a typical particle separation. The suspension only behaved like an effective fluid on a longer time scale, of the order $\tau_{\phi}>4$. To be more precise on time scales for which the VACF of an isolated particle could be described by its asymptotic decay. At low densities, where the suspension viscosity $\nu_{\phi}$ is only slightly different from the fluid viscosity $\nu_{0}$, this is almost equivalent to the

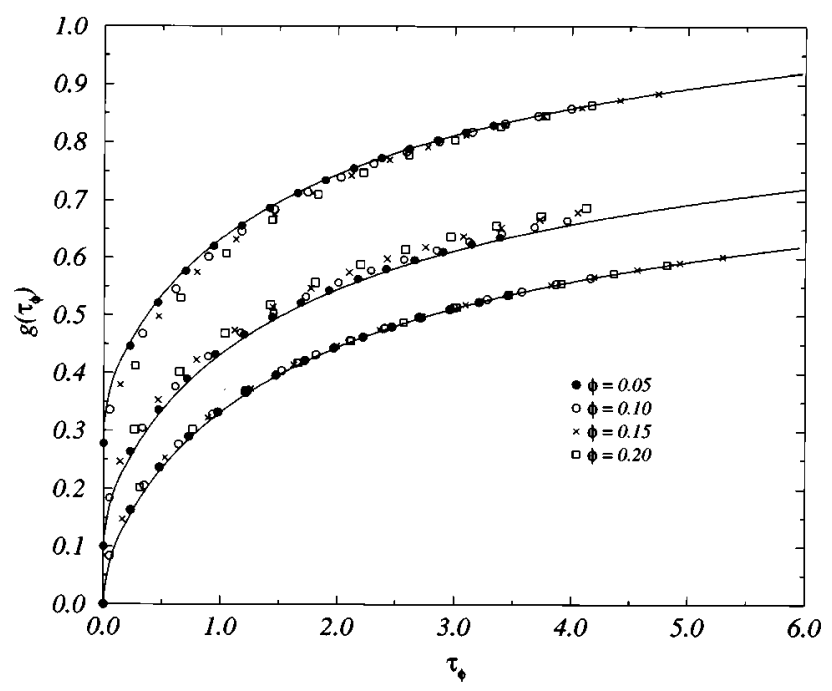

FIG. 6. The scaled mean-squared displacement $g\left(\tau_{\phi}\right)$ as a function of the reduced time $\tau_{\phi}$. The upper scaling is the scaling we propose in the text (displaced by 0.3 in the $y$ direction). The middle scaling is the scaling proposed by Zhu et al. using our calculated value for the suspension viscosity (displaced by 0.1 in the $y$ direction). The lower scaling is the scaling proposed by Zhu et al. but with the suspension viscosity as a free parameter. The data were obtained with spheres of effective radius 2.46. In each case the solid line is the single-particle result.

time taken for vorticity to diffuse a particle diameter. At shorter times the behavior of a particle in the suspension cannot be described by rescaling the isolated particle result. At higher viscosities, where the suspension viscosity is significantly higher than the fluid viscosity, this time becomes

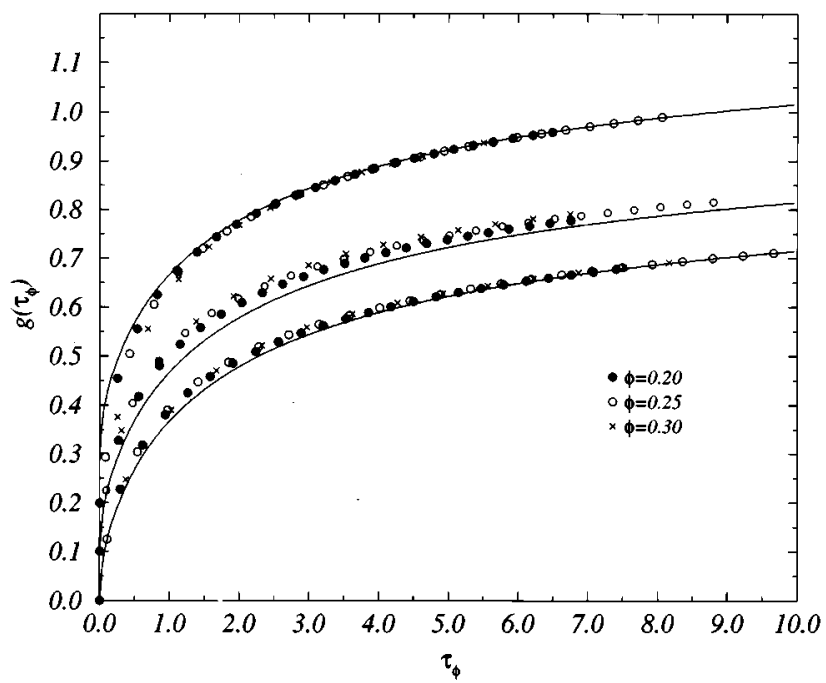

FIG. 7. The scaled mean-squared displacement $g\left(\tau_{\phi}\right)$ as a function of the reduced time $\tau_{\phi}$. The upper scaling is the scaling we propose in the text (displaced by 0.2 in the $y$ direction). The middle scaling is the scaling proposed by Zhu et al. using our calculated value for the suspension viscosity (displaced by 0.1 in the $y$ direction). The lower scaling is the scaling proposed by Zhu et al. but with the suspension viscosity as a free parameter. The data were obtained with spheres of effective radius 4.20. In each case the solid line is the single-particle result. 
somewhat less than the time taken to diffuse a particle diameter. However, the interparticle separations also start to become smaller. We therefore conclude that our results are consistent with the hypothesis that hydrodynamic interactions develop by the diffusion of transverse momentum through the fluid. We do not seem to need a "superfast" speed of sound mechanism.

We can gain further insight into this by considering the speed of sound in our simulations. First we can define a characteristic time based on the time taken for vorticity to diffuse a particle radius $\tau_{\nu}=a^{2} / \nu$. We can also define a second characteristic time based on the time taken for a sound wave to propagate a particle radius $\tau_{s}=a / c_{s}$. In our simulations the ratio $\tau_{s} / \tau_{\nu}$ is equal to about 0.1 for the spheres with radius 2.5 and 0.2 for the spheres of radius 4.5 (this is the reason we generally plotted the results obtained for the two sizes of spheres separately, otherwise we would not have been comparing like with like). In a typical colloid studied experimentally the ratio should be more like 0.01 , but our simulations basically satisfy the condition $\tau_{s} \ll \tau_{\nu}$, i.e., that sound propagates much faster than vorticity. At a volume fraction of 0.2 we used both sphere radii. The only difference between the two calculations is that in one case $(a=2.5)$ we have $\tau_{s} / \tau_{\nu}=0.1$ and in the second $(a=4.5)$ we have $\tau_{s} / \tau_{\nu}=0.2$. We can find no convincing evidence that the time required for effective-fluid behavior to be observed differs in the two cases. This is despite the fact that the ratio $\tau_{s} / \tau_{\nu}$ differs by a factor of two. However, if we ask the question, are the results the same? then the answer is: not quite. If we look at the scaled VACF for the particles with radius 2.5 at a volume fraction of 0.2 (Fig. 4) and compare it with the equivalent plot for the spheres of radius 4.5 (Fig. 5) then at short times there is a perceptible difference. For instance, at a time $\tau_{\phi} \sim 1$ the scaled VACF for the larger spheres (in the system with the proportionately lower speed of sound) is actually less than the isolated particle VACF. This never appears to be the case for the smaller spheres. So our results tend to suggest that sound wave propagation may influence the manner in which effective-fluid behavior is reached, but not the time scale.

We conclude by noting that there is also a rather fundamental objection to the suggestion that hydrodynamic interactions propagate with the speed of sound. In our discussion of the scaling of the VACF we introduced a function which quantified the deviation of the scaled VACF from the isolated particle VACF. The integral of this function was defined by the suspension viscosity and the suspension (shorttime) diffusion coefficient. Neither of these quantities depend on the speed of sound so neither can the total deviation between the two functions. In other words, no matter what the speed of sound, the scaled VACF in a suspension differs from the scaled single-particle VACF by an amount that is independent of the speed of sound.

\section{ACKNOWLEDGMENTS}

Thanks to Jaydeb Chakrabarti, Simon de Leeuw, and Andrew Masters for their critical reading of the manuscript. The work of the FOM Institute is part of the scientific program of FOM and is supported by the Nederlandse Organisatie voor Wetenschappelijk Onderzoek (NWO). This work was also supported by SON. Computer time on the CRAY-C98/4256 at SARA was made available by the Stichting Nationale Computer Faciliteiten (N.C.F.).

\section{APPENDIX}

As we showed in the argument leading up to Eq. (12), the scaling proposed by Zhu et al. for the time-dependent diffusion coefficient $D(t)$ corresponds to the following scaling for the $\operatorname{VACF} f_{\phi}\left(\tau_{0, \phi}\right)$ :

$$
f_{\phi}\left(\tau_{0, \phi}\right)=\frac{9}{2} \frac{C_{v, \phi}\left(\tau_{0, \phi}\right)}{C_{v}(0)} \frac{D_{S E, \phi}}{D_{\phi}} .
$$

If we follow the same procedure as Zhu et al. but, instead of dividing $D(t)$ by the suspension viscosity $D_{\phi}$ we divide by the Stokes-Einstein diffusion coefficient then, the new scaled $\operatorname{VACF} f_{\phi}^{*}\left(\tau_{0, \phi}\right)$ is

$$
f_{\phi}^{*}\left(\tau_{0, \phi}\right)=\frac{9}{2} \frac{C_{v, \phi}\left(\tau_{0, \phi}\right)}{C_{v}(0)}
$$

compared with the scaled VACF for a single particle $f\left(\tau_{0}\right)$ which was given by

$$
f\left(\tau_{0}\right)=\frac{9}{2} \frac{C_{v}\left(\tau_{0}\right)}{C_{v}(0)} .
$$

Effective-fluid behavior corresponds to the VACF in the suspension being the same as the VACF for a single particle in a fluid with the suspension viscosity, i.e.,

$$
C_{v, \phi}\left(\tau_{0, \phi}\right)=C_{v}\left(\tau_{0}\right)
$$

corresponding to

$$
f_{\phi}^{*}\left(\tau_{0, \phi}\right)=f\left(\tau_{0}\right) .
$$

However, remember that to the scaling leading to $f_{\phi}^{*}\left(\tau_{0, \phi}\right)$ involved dividing by the Stokes-Einstein diffusion coefficient and not the true short-time diffusion coefficient. This means that, while the integral over all times of the right hand side of Eq. (A5) is equal to unity, the integral over all times of the left hand side is equal to $D_{\phi} / D_{S E, \phi}$. In other words Eq. (A5) cannot apply over all times so neither can effectivefluid behavior. One way around this problem is to accept that $C_{v, \phi}\left(\tau_{0, \phi}\right)$ cannot be equal to the single-particle function $C_{v}\left(\tau_{0, \phi}\right)$ over all times, but speculate that it is at long times. In this case we can write the scaled VACF in a suspension as the sum of the scaled single-particle VACF plus some "deviation" function $\delta_{\phi}\left(\tau_{0, \phi}\right)$, i.e.,

$$
C_{v, \phi}\left(\tau_{0, \phi}\right)=C_{v}\left(\tau_{0, \phi}\right)+\delta_{\phi}\left(\tau_{0, \phi}\right) .
$$

If, on some time scale, the deviation function has decayed to zero, the suspension will behave like an effective fluid and the scaled function will become

$$
f_{\phi}^{*}\left(\tau_{0, \phi}\right)=\frac{9}{2} \frac{C_{v}\left(\tau_{0, \phi}\right)}{C_{v}(0)}=f\left(\tau_{0, \phi}\right) .
$$

From Eq. (5), notice that the corresponding scaled timedependent diffusion coefficient $g_{\phi}\left(\tau_{0, \phi}\right)$ involves a time in- 
tegral of Eq. (A6), so the terms involving $\delta_{\phi}\left(\tau_{0, \phi}\right)$ will all approach a constant if $\delta_{\phi}\left(\tau_{0, \phi}\right)$ approaches zero. If this is the case then we will have

$$
\begin{aligned}
g_{\phi}\left(\tau_{0, \phi}\right)= & \int_{0}^{\tau_{0, \phi}} f\left(\tau_{0, \phi}^{\prime}\right) d \tau_{0, \phi}^{\prime} \\
& +\frac{1}{\tau_{0, \phi}} \int_{0}^{\tau_{0, \phi}} \tau_{0, \phi}^{\prime} f\left(\tau 0, \phi^{\prime}\right) d \tau_{0, \phi}^{\prime}+\text { const, }
\end{aligned}
$$

[1] A. Einstein, Ann. Phys. (N.Y.) 17, 549 (1905).

[2] A. Einstein, Investigations on the Theory of Brownian Movement (Dover, New York, 1956).

[3] A. Einstein, Ann. Phys. (N.Y.) 34, 591 (1911).

[4] N.G. van Kampen, Stochastic Processes in Physics and Chemistry (North-Holland, Amsterdam, 1981).

[5] B.J. Alder and T.E. Wainwright, Phys. Rev. A 1, 18 (1970).

[6] M.H. Ernst, E.H. Hauge, and J.M.J. van Leeuwen, Phys. Rev. Lett. 25, 1254 (1970).

[7] J.R. Dorfman and E.G.D. Cohen, Phys. Rev. Lett. 25, 1257 (1970).

[8] Y.W. Kim and J.E. Matta, Phys. Rev. Lett. 31, 208 (1973).

[9] P.D. Fedele and Y.W. Kim, Phys. Rev. Lett. 44, 691 (1980).

[10] G.L. Paul and P.N. Pusey, J. Phys. A 14, 3301 (1981).

[11] Chr. Morkel, Chr. Gronemeyer, W. Gläser, and J. Bosse, Phys. Rev. Lett. 58, 1873 (1987).

[12] J.J. Erpenbeck and W.W. Wood, Phys. Rev. A 32, 412 (1985).

[13] M.A. van der Hoef and D. Frenkel, Phys. Rev. A 41, 4277 (1990).

[14] A.J.C. Ladd, J. Fluid Mech. 271, 311 (1994).

[15] M. Hagen, C.P. Lowe, and D. Frenkel, Phys. Rev. E 51, 4287 (1995).

[16] C.P. Lowe, D. Frenkel, and A.J. Masters, J. Chem. Phys. 103, 1582 (1995). where the first two terms are just the single-particle scaling with $\tau_{0, \phi}$ replacing $\tau_{0}$. In other words, if the suspension behaves like an effective fluid then, using the modified scaling we have proposed, the time-dependent diffusion coefficient, plotted in terms of the suspension viscosity, will differ from the single-particle function by a constant. The value of this constant is most conveniently chosen to be $1-D_{\phi} / D_{S E, \phi}$, in which case $g_{\phi}\left(\tau_{0, \phi}\right)$ will have an asymptotic value of unity, independent of $\phi$.
[17] E.H. Hauge and A. Martin-Löf, J. Stat. Phys. 7, 259 (1973).

[18] J.X. Zhu, D.J. Durian, J. Müller, D.A. Weitz, and D.J. Pine, Phys. Rev. Lett. 68, 2559 (1992).

[19] C.W.J. Beenakker and P. Mazur, Physica A 120, 388 (1983).

[20] C.W.J. Beenakker and P. Mazur, Physica A 128, 48 (1984).

[21] P.N. Pusey and W. van Megen, J. Phys. (Paris) 44, 258 (1983).

[22] A.J.C. Ladd, J. Chem. Phys. 93, 3484 (1990).

[23] M.H. Kao, A.G. Yodh, and D.J. Pine, Phys. Rev. Lett. 70, 242 (1993).

[24] A.J.C. Ladd, Phys. Rev. Lett. 70, 1339 (1993).

[25] P. Español, M.A. Rubio, and I. Zúñiga, Phys. Rev. E 51, 803 (1995).

[26] P. Español, Physica A 214, 185 (1995).

[27] S.T. Milner and A.J. Liu, Phys. Rev. E 48, 449 (1993).

[28] B. Cichocki and B.U. Felderhof, Physica A 211, 25 (1994).

[29] B. Cichocki and B.U. Felderhof, Phys. Rev. E 51, 5549 (1995).

[30] U. Frisch, B. Hasslacher, and Y. Pomeau, Phys. Rev. Lett. 65, 1505 (1986).

[31] G.R. McNamara and B.J. Alder, in Microscopic Simulation of Complex Hydrodynamic Phenomena, edited by M. Mareschal and B.L. Holian (Plenum, New York, 1992).

[32] U. Frisch, D. d'Humières, B. Hasslacher, P. Lallemand, Y. Pomeau, and J.-P. Rivet, Complex Syst. 1, 649 (1987).

[33] A.J.C. Ladd, J. Fluid. Mech. 271, 285 (1994).

[34] A.J.C. Ladd and D. Frenkel, Phys. Fluids A 2, 1921 (1990). 